MEDICAL EDUCATION

\title{
Communication skills training in postgraduate medicine: the development of a new course
}

\author{
J Dacre, J Richardson, L Noble, K Stephens, N Parker
}

Postgrad Med J 2004;80:71 1-715. doi: 10.1136/pgmj.2004.022129

It has long been accepted that communication is of central importance in healthcare, and a core aspect of clinical competence. Many educational institutions and Royal Colleges now reflect this and consider communication skills a priority in postgraduate examination. The new examination "Practical Assessment of Clinical and Examination Skills" has replaced the Royal College of Physicians MRCP part 2 clinical and oral examination. This examination now consists of five clinical stations, two of which focus on communication skills. A short course for postgraduate trainees has been designed to address the communication skills requirements of the part 2 clinical examination. The aims, development, and content of the course are described. Emphasis is placed on candidates practising skills with patients and receiving feedback during the course. Evidence suggests that practice with feedback is an essential ingredient of communication skills courses, and is more effective than other methods such as observing experts or video examples, or simply discussing issues in communication. Results of a preliminary evaluation indicate that the course was perceived as valuable by candidates and that the aims, format, and content were appropriate. Although the preliminary evaluation was largely positive, it could be argued that the acid test of the effectiveness of a course is an objective evaluation of skills, observed before and after the course, a development that is being considered for future evaluation of the course. Recommendations for applying this type of training to postgraduate trainees in any branch of medicine are given.

See end of article for authors' affiliations

Correspondence to: Professor Jane Dacre, Academic Centre for Medical Education, 4th Floor, Holborn Union Building, Archway Campus, Highgate Hill, London N19 5LW, UK; j.dacre@chime.ucl.ac.uk

Submitted 26 March 2004 Accepted 28 May 2004

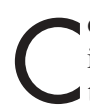
ommunication skills training has become increasingly important in medical education worldwide. ${ }^{1}$ The effectiveness of specific teaching methods, which include the use of practice with simulated patients and feedback to trainees, has been established in both undergraduate and postgraduate training in the UK, USA, Europe, and Australia. ${ }^{2-4}$ Communication skills teaching has now become commonplace in UK medical undergraduate curricula following recommendations published by the General Medical Council. ${ }^{5}$

It has long been accepted that communication is of central importance in healthcare, and a core aspect of clinical competence. ${ }^{6}$ It is also clear that good communication skills in medical practice are not innate, can be learned, and can always be enhanced. ${ }^{7}$ Good doctor-patient communication leads to greater patient satisfaction and makes a significant difference to patient health outcomes. ${ }^{8}$ However, the majority of complaints to UK hospital trusts can be traced back to poor communication.'

The General Medical Council has given guidance to enhance doctors' communication skills in its document Good Medical Practice. ${ }^{10}$ This states that good communication involves listening to patients, giving information, sharing information with patients' partners and relatives, and communicating with colleagues. While evidence suggests that doctors can be taught and do learn from training in communication skills, training at the postgraduate level in the UK has lagged behind the developments in undergraduate teaching. In line with the medical education adage "assessment drives learning", trainees' attendance at courses and training sessions tends to reflect their immediate needs to fulfil the requirements of postgraduate examinations. The introduction of communication skills assessment into postgraduate examinations can therefore provide an opportunity to encourage trainees to reflect on and enhance their own communication.

The Royal College of Physicians has considered communication skills as a priority in the redevelopment of their part 2 clinical examination. The new examination "Practical Assessment of Clinical and Examination Skills" (PACES) replaced the MRCP part 2 clinical and oral examination in June 2001, and consists of five clinical stations, two of which concentrate on communication skills. These are a history taking station (information gathering) and a communication skills and ethics station (information giving). During these stations candidates are asked to conduct a consultation with a patient or relative for a period of 14 minutes followed by one minute of reflection. The candidates are then asked questions by the examiner about the clinical content and communication issues involved in the presented scenario for a final five minutes.

In this article, we describe a communication skills course that has been piloted on candidates preparing for the PACES examination and is not provided elsewhere in the UK to date. The development of this course was supported by the Fellowship of Postgraduate Medicine, which has had a longstanding interest in the development of communication skills training of postgraduate doctors. 
The aim of this report is to share the experience of developing and implementing a course in this area, and to demonstrate the generalisability of this type of teaching to other branches of medicine.

\section{COURSE AIMS}

Our aim was to focus candidates on achieving a pass level in the communication skills stations of the PACES examination (stations 2 and 4), according to the criteria published by the Royal College of Physicians. ${ }^{11}$ The course was therefore designed to improve participants' skills in the basic communication techniques required for consultations with patients and relatives, as appropriate to the training grade of the participants (that is, UK senior house officer level). Although the course focused on the PACES examination, the teaching was designed to be extrapolated to other areas within the healthcare arena.

\section{COURSE DEVELOPMENT}

We endeavoured to set up a course that would be accessible to candidates in the local trust as well as applicants from other areas. A working group was therefore convened which comprised a medical education expert, a clinical director of the trust, both of whom were physicians and experienced examiners at postgraduate level, a communication skills expert, and an ethics expert.

The working group met on several occasions and agreed on the following key features of the course.

\section{Course design}

A two day course was chosen to allow a sufficient intensity of practice, while being short enough to enable candidates to protect the time required. A single block of time was chosen, rather than shorter sessions over a longer period, to facilitate attendance by non-local trainees.

An emphasis was placed on candidates practising skills with patients and receiving feedback during the course. Evidence suggests that practice with feedback is the essential ingredient of communication skills courses, ${ }^{2-4}$ and is more effective than other methods such as observing experts or video examples, or simply discussing issues in communication. It was decided to place participants in small groups to enable each to have the opportunity to conduct consultations and observe peers' consultations. The method of feedback chosen was the standard approach taken in communication skills training, which comprises self reflection from the candidate to encourage insight into their own skills and comments from observers (peers, tutors, and simulated patients). Feedback was given broadly based on Pendleton's rules. ${ }^{12}$ Using this method, candidates are asked what they did well, followed by observers' positive comments. They are then asked to reflect on what they could have improved on, followed by suggestions from the observers.

To address the potential areas covered in the examination, it was decided to allocate each half day of the course to a specific topic. These were history taking (information gathering), information giving, breaking bad news, and situations involving ethical issues. For each session, a selection of consultations were chosen which the working group felt were representative of situations that candidates could encounter in normal clinical practice. It was considered important that candidates should be exposed to situations that would trigger their normal repertoire of clinical and communication skills, rather than providing extreme situations which would cause them to have to "act" how they might respond. To this end, the case scenarios used were written by the course organisers based on real, anonymised cases illustrating a particular clinical situation or dilemma.
Box 1: Scenario 1

Mr Jones, 20 years old, accident and emergency department.

\section{Instructions to doctor}

Mr Jones has been admitted to casualty having taken 50 paracetamol tablets and two pints of bitter. The overdose followed a row with his girlfriend during which she walked out and left him. A couple of hours after taking the pills, he rang a friend who brought him into casualty. Two hours after that he had a stomach pump. It is now $2.00 \mathrm{am}$. His blood levels of paracetamol are still very high.

There is a high risk of liver damage and that Mr Jones will die without further treatment. Treatment is an antidote called acetylcysteine which is given by means of a drip. Mr Jones will have to stay in overnight, perhaps for two nights, and have his blood levels checked daily. He will be kept under close observation for signs of liver failure (going yellow, swelling up, and becoming comatose). If he refuses to stay in the hospital, the doctor can invoke a section of the Mental Health Act as Mr Jones is at risk of harming himself as a result of self poisoning. However, a voluntary admission is preferable both in terms of administrative time and maintaining good rapport.

Instructions to simulated patient

Mr Jones is now tired and irritable because he wants to go home. His girlfriend has arrived and he is content that things have been patched up with her. He is also very worried that if he doesn't go home with her she may well find comfort elsewhere, as the cause of the row had been that she was becoming overfriendly with a mate. He knows the casualty staff think he is a waste of space (they have been quite rude to him because he "has brought this on himself"). He doesn't want to stay in hospital any longer.

\section{Patients}

In order to balance the twin goals of realism and exposure to more complex situations (for example, breaking bad news), it was decided to use a combination of real and simulated patients. Real patients were chosen for the history taking consultations, to ensure that the depth of detail in the histories was sufficient for a postgraduate course, and to emphasise to candidates that the course was focusing on their normal response to real patients. Simulated patients were used for the sessions focusing on the communication skills and ethics station, as they were able to demonstrate a range of difficult and/or emotional situations, and were able to supply detailed feedback on the experiences of the character required by the scenario.

\section{Tutors}

In order to ensure that both the physician and the communication skills perspectives were represented-and seen by the candidates to be represented-two tutors were allocated per group, one physician and one communication skills expert.

\section{Standard expected}

To set a level for expected performance in the consultations, it was decided to use the PACES marking schedules and anchor statements ${ }^{11}$ as a guide throughout the course.

\section{Format}

In order to familiarise candidates with the format of the examination, it was decided that a similar format would be used in the sessions on the course-that is, candidates would 


\section{Box 2: Scenario 2}

Mr Arnold, 38 years old, accident and emergency department.

\section{Instructions to doctor}

Mr Arnold is the husband of Elaine Arnold, a 35 year old woman admitted to hospital earlier that day with acute asthma. She was brought into the hospital by ambulance; the ambulance having arrived within 20 minutes of the original call. Mrs Arnold had had previous admissions with asthma and was known to dislike using inhalers. Within 30 minutes of her arrival today, while awaiting chest radiography, she had a cardiac arrest. Exactly why this happened is not clear. Resuscitation attempts were not successful. Mr Arnold has arrived and does not yet know that his wife has died.

\section{Instructions to simulated relative}

Mr Arnold is irritated at having been called from work because it has interfered with his plans. His wife has had many asthma attacks - what is all the fuss about now? When he is told about his wife's death he at first refuses to believe it. When he is convinced that she has died he may express any of the following emotions:

- Continuing disbelief.

- Concern for their children, aged 7 and 5, who are at school.

- Suspicion that someone has not done their job.

- Guilt because he knows his wife was unwell when he left for work and he thought she was "playing for sympathy" after an argument.

- Anger with his wife-why didn't she take her medicines properly?

- Anger with the hospital/medical profession-how could a young woman die from such a trivial illness?

- Fear that "they" might mutilate her body with a postmortem.

Exactly which of these emotions predominates will depend on how the doctor gives him information about his wife's death.

be given five minutes to read the information about the task, followed immediately by a 14 minute consultation, followed by one minute of reflection, then five minutes of viva questioning. Detailed feedback would then follow.

\section{COURSE PROCEDURE}

Tutors were recruited from local sources-for example, the medical school and the local trust. They were given guidance on the format of the examination, and the aims, format, content, and time frame of the course. Pairs of tutors were encouraged to develop their own style when running the sessions, so that the participants came across a variety of effective teaching approaches.

Candidates were allocated to groups (4-6 per group) with two tutors. Both candidates and tutors were provided with the PACES marking schedules and anchor statements. Apart from brief plenary introductory and feedback sessions, candidates remained in the same groups throughout the course. Groups rotated among pairs of tutors.

During each half day session the candidates were instructed around three or four scenarios designed to raise specific communication skills issues or ethical dilemmas. A total of 45 minutes was allocated for each case scenario or real patient case, which included preparation, practice, and feedback. Candidates performed the consultations "goldfish bowl style" in the same room as the other participants and tutors. As the aim of the course was to focus on communication skills rather than clinical skills per se, any complex medical information which might be beyond the level of clinical competence expected at senior house officer level was provided to candidates. Examples of scenarios used are shown in boxes 1 and 2 .

\section{EVALUATION OF THE COURSE}

For the purposes of a preliminary evaluation, candidates' and tutors' perceptions of the course were obtained. The course has run four times, with a total of 87 candidates attending. Of these, 76 candidates filled in an evaluation questionnaire (response rate of $87 \%$ ).

The majority of candidates had trained outside the UK and English was a second language. A minority were UK based trainees. In the later courses many candidates reported that they had previously failed examinations and were due to re-sit.

\section{Candidates' perceptions of the course}

Feedback from the course was extracted from a questionnaire where candidates were asked to rate the value of the course (see figs 1-3 for feedback from one of the courses; 22 of 24 candidates completed the evaluation forms). The responses indicated that the majority of candidates found it extremely valuable. All sessions were reported to be useful, although the session discussing ethical dilemmas was thought to be marginally more useful than the other topics. Additional comments from candidates indicated that they found teaching on how to plan and structure the interview particularly useful, and in particular how to use the five minutes of planning time before the consultation. Candidates reported that they wanted honest feedback on both good and poor aspects of their performance, and that they liked the tutors who gave this. Some said that all sessions were useful, but they learned most from their mistakes. Having the opportunity to practice was felt particularly valuable. Many candidates were focused on their preparation for the PACES examination and appreciated those sessions where tutors were able to reproduce examination conditions. Candidates agreed that the case scenarios were realistic.

"One of the best courses I have ever done-incredibly useful both for clinical practice and the exam".

Candidates found sessions least useful when not many of them were able to attempt consultations (which was either due to larger group size or prolonged discussions resulting from a candidate's performance in a previous scenario). When candidates performed badly, they sometimes felt that tutors did not tackle all of the aspects that needed to change. Some candidates felt that more scenarios overall were needed.

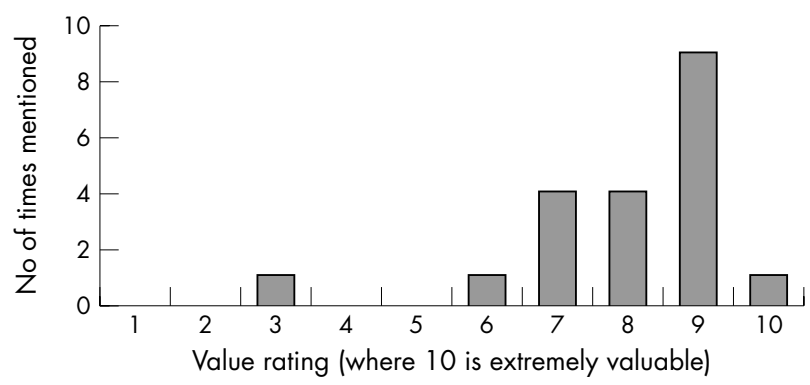

Figure 1 How valuable was the course overall? 


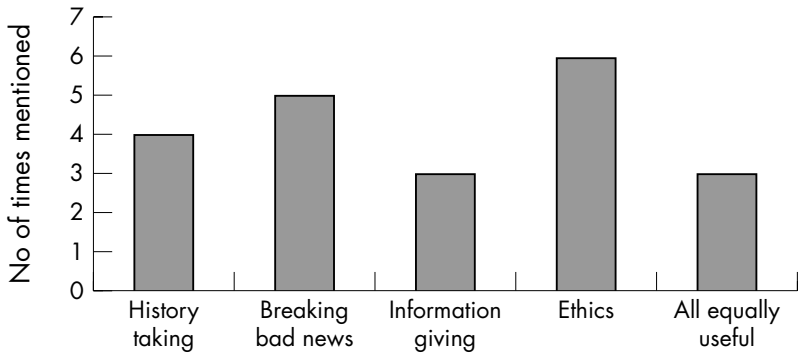

Figure 2 Which session was most valuable and why?

Candidates reported that they would have liked more scenarios or smaller groups, a longer course, more theory/ teaching/lectures, more opportunities to consider ethical issues, the opportunity for feedback with video review, and examination conditions reproduced closely in all the sessions (for example, timing of each section).

In general, all candidates were satisfied with the tutors, who were reported to be thorough and very constructive in their criticism. Some candidates commented that different tutors approached issues in quite different ways: some were supportive facilitators, and others used more aggressive or confrontational styles similar to that traditionally expected in oral examinations. Those tutors who were able to replicate the examination viva style for part of the session were rated highly. One candidate commented particularly on the balance between physicians and communication skills experts, which was felt to work well.

\section{Tutors' perceptions of the course}

Tutors were asked for their feedback informally. Most reported that they enjoyed working with the candidates in small groups and co-facilitating with the other tutors. Working with the simulated patients was generally reported to be positive, particularly those who gave effective and constructive feedback. Working with real patients was also found to be generally positive, although some patients were not able to provide a consistent story, or were too experienced in giving their history to respond to the different skills demonstrated by candidates. Although there was no formal assessment of candidates' skills during the course, tutors felt that overall the standard of performance of communication tasks improved significantly over the two days, and they noticed candidates putting suggestions into practice in subsequent consultations.

Several points of good practice in conducting a consultation were highlighted by tutors during the first four courses, which it was felt might be included as preparation for the course or as a handout. These were:

- The importance of reflection before a consultation in order to form a clear agenda of the overall aims of the consultation and prepare questions. (This is encouraged by the five minutes of preparation time in the PACES examination.)

- Checking the patient's name as the consultation begins. Starting with an open question.

- The need for effective questioning: using a mixture of open and closed questions, structuring the questions carefully, and exploring each area in full before moving on. Taking care not to interrogate patients.

- Avoiding the use of medical language and checking at each stage that patients have understood what is being said.

- Ensuring that the doctor does not push his or her own agenda.

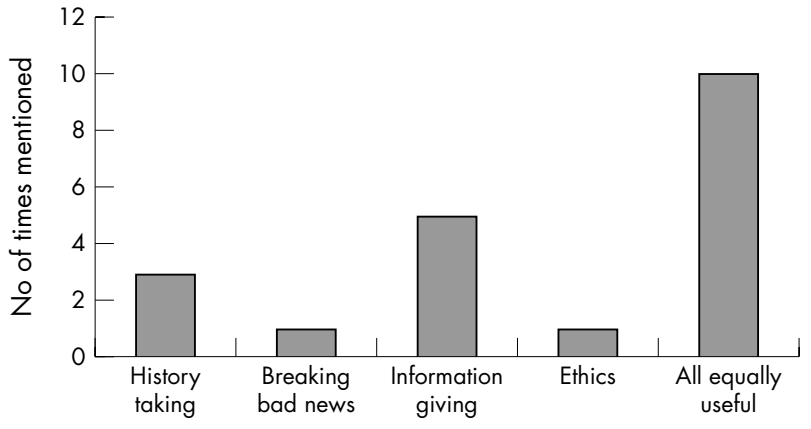

Figure 3 Which session was least valuable and why?

- Allowing patients time to finish speaking, using verbal and non-verbal cues to make it clear that the doctor is listening. Responding to the information the patient has given to show that this has been heard and understood. Using careful interjections to redirect the interview if necessary.

- Avoiding premature closure (finishing very quickly). There should be a summary-for example, recapping decisions which have been made, and agreement of an immediate plan for the next step.

\section{DISCUSSION}

We have described the development, content, and implementation of a course on communication skills for postgraduate physicians. Preliminary feedback from candidates suggests that the course is perceived as valuable and that the format and content are appropriate. In a course of this kind a balance must always be struck between competing demands-for example, whether to have a smaller number of scenarios and comprehensively address the issues raised, or run shorter scenarios so candidates have more opportunity to practise. Comments from candidates suggesting improvements to the course indicated that often contradictory changes were desired, which suggests that the course is in general achieving a balance. The reproduction of examination conditions, was however, universally reported as helpful. Interestingly, candidates did not comment on the use of simulated patients or suggest that conducting observed consultations in a group felt unrealistic, a common criticism levelled at communication skills teaching. In contrast, candidates reported that both the history taking and simulated consultations were realistic and valuable. From a practical point of view, the use of simulated patients was a major financial cost, but this was offset by calculating an optimum group size and course fee.

The majority of participants attending the course were not UK graduates and/or had already failed the PACES examination previously, suggesting that this subgroup of doctors felt particularly in need of preparation. It was striking that the list of skills drawn up by tutors that candidates needed to focus on is no different to the skills taught at undergraduate level, suggesting either that the candidates attending had not had this type of teaching before, or that they had not developed the skills during their clinical work, or simply that the key features of good communication are common to all consultations. The candidates particularly reported that they wanted more formal teaching about ethical issues, which may reflect the relative newness of this subject in medical curricula in general.

Although the preliminary evaluation was largely positive, it could be argued that the acid test of the effectiveness of a course is an objective evaluation of skills, observed before and 
after the course. This is a development that we are considering for future evaluation of the course. An alternative approach, to compare the pass rates of the course participants to pass rates in PACES overall, was rejected as an evaluation strategy due to the self selection of candidates attending the course. From the point of view of considering the effectiveness of the course at this point, therefore, we can say that the course was designed to include features of communication skills training that are known to be effective from previous research, and that candidates reported that the course was valuable in helping them to learn communication skills.

We believe that this is an important part of postgraduate training and more courses of this type would improve the standard and quality of communication skills of doctors in the UK. We would like to encourage the development of similar courses for other clinical specialties. Our recommendations for key features of such courses are: (1) linking training to assessment, due to the importance of assessment as a motivating factor, (2) setting a standard according to professional guidelines (for example, General Medical Council recommendations or Royal College examination criteria), (3) designing a course that reflects the evidence about effective ingredients of communication skills training, available from the literature, particularly focusing on practice with feedback, (4) ensuring that the level and content are appropriate to the current level of the course participants (so that participants do not have to "act") and that the content reflects routine, realistic scenarios (rather than extreme and unusual situations), and (5) considering the balance between desired features of the course and practicalities (such as cost, course timing and length, number of participants, and type of patients). We hope that our experience of developing this course, which has been very positive, will encourage others to do likewise.

\section{ACKNOWLEDGEMENTS}

We would like to thank Richard Meakin for providing advice about the ethical content of the course.

\section{Authors' affiliations}

J Dacre, J Richardson, L Noble, K Stephens, Academic Centre for Medical Education, Royal Free and University College Medical School, London, UK

N Parker, Whittington Hospital NHS Trust, London, UK

\section{REFERENCES}

1 Simpson M, Buckman R, Stewart M, et al. Doctor-patient communication: the Toronto consensus statement. BMJ 1991;303:1385-7.

2 Aspegren K. Teaching and learning communication skills in medicine: a review with quality grading of articles. Medical Teacher 1999;21:563-70.

3 Hulsman RL, Ros WJG, Winnubst JAM, et al. Teaching clinically experienced clinicians communication skills: a review of evaluation studies. Med Educ 1999;33:655-68.

4 Kurtz SM, Laidlaw T, Makoul G, et al. Medical education initiatives in communication skills. Cancer Prevention and Control 1999;3:37-45.

5 General Medical Council. Tomorrow's doctors: recommendations for undergraduate medical education. London: GMC, 1993.

6 Association of American Medical Colleges. Contemporary issues in medicine: communication in medicine. Washington, DC: AAMC, 1999.

7 Maguire $P$, Pitceathly $C$. Key communication skills and how to acquire them. BMJ 2002;325:697-700.

8 Stewart $M$. Effective physician-patient communication and health outcomes: a review. CMAJ 1995;152:1423-33.

9 Richards T. Chasms in communication. BMJ 1990;301:1407-8.

10 General Medical Council. Good medical practice. 3rd Ed. London: GMC, 2001.

11 Royal College of Physicians. MRCP(UK) clinical guidelines. London: RCP, 2002.

12 Pendleton D, Schofield T, Tate P, et al. The consultation: an approach to teaching and learning. Oxford: Oxford University Press, 1984. 